\title{
Dust Evolution in Protoplanetary Disks
}

Thomas Henning, Wolfgang Schmitt, Hubert Klahr, and Rastislav Mucha

Max Planck Society, Research Unit "Dust in Star-forming Regions" Schillergäßchen 2-3, 07745 Jena, Germany

\begin{abstract}
The evolution of dust particles in circumstellar disk-like structures around protostars and young stellar objects is discussed. We especially consider the coagulation of grains due to collisional aggregation and the influence of this process on the optical properties of the particles. These dust opacities are important for both the derivation of the circumstellar dust mass from submillimetre continuum observations and the dynamical behaviour of the disks.

We present first results of a numerical study of the coagulation of dust grains in a turbulent protoplanetary accretion disk described by a time-dependent one-dimensional (radial) "alpha" model. The influence of grain opacity changes due to dust coagulation on the dynamical evolution of a protostellar disk is investigated. In addition, we consider the grain motion in two-dimensional disks.
\end{abstract}

\section{Introduction}

Although the formation of planetesimals in the protoplanetary accretion disk by accumulation of presolar particles or nebula condensates is generally accepted, two completely different scenarios were proposed: (1) Safronov (1969) and Goldreich \& Ward (1973) attributed the formation of planetesimals to gravitational instabilities in a thin midplane dust layer of the disk. For this spontaneous process, the physical or chemical properties of the dust grains are unimportant, but the conditions in the nebula gas are crucial: the nebula gas has to be quiescent to allow the sedimention of the dust grains toward the disk's midplane and hence to form a dense dust layer. (2) However, it was argued that the solar nebula was turbulent, and such turbulence would alter the above scenario considerably (see, e.g., Völk et al. 1980). Even low random gas velocities are sufficient to prevent the dust layer from becoming gravitationally unstable (Weidenschilling 1988). In this case, larger particles may form by the coagulation or aggregation of colliding grains. Mutual collisions between dust particles are caused by the turbulence of the nebula gas or by differential settling and drifts of unequally sized grains. Using the turbulence-induced velocities calculated by Völk (1980), Mizuno et al. (1988) and Mizuno (1989) solved the coagulation equation and calculated the time evolution of the dust mass spectra and the resulting Rosseland mean opacities at a certain disk radius. Ruden \& Pollack (1991) considered the global evolution of protostellar nebulae assuming thermal convection as the only 
source of turbulence and hence viscosity. In their model, however, they neglect the growth of the dust grains. Weidenschilling \& Cuzzi (1993) calculated grain velocities in the solar nebula and deduced size spectra for the dust aggregates. They found that at least for an earlier stage of particle growth ( $\mu \mathrm{m}$ to $\mathrm{m}$ sizes), sticking and coagulation of particles are the most likely processes responsible for the rapid growth of pre-planetesimal dust.

Within this approach, fundamental problems are still unresolved: There is no self-consistent treatment of the disk and dust evolution including the optical properties of the particles available. In addition, one needs a two-dimensional treatment of the process including vertical and radial velocities. The question of the influence of magnetic fields on charged grains is not answered. Furthermore, a more comprehensive knowledge of the physics of the coagulation process is needed.

\section{Coagulation in a $1 \mathrm{D}$ Disk}

\subsection{Model Description}

For the numerical simulation of the evolution of dust grains in a turbulent protoplanetary accretion disk, we developed a time-dependent one-dimensional (radial) "alpha" model (see Ruden \& Pollack 1991). By calculating simultaneously the integrodifferential equation of the coagulation process (e.g. Mizuno et al. 1988, Mizuno 1989), the growth of dust grains due to collisional aggregation caused by relative velocities between the particles is taken into consideration. The relative velocities may be induced by thermal motion, turbulence, and drift motion. As initial dust distribution, we use a mass spectrum typical for the diffuse interstellar medium. Coagulation of particles changes the opacities, and the opacities on their part will influence the dynamical evolution of the protoplanetary accretion disk. The scheme of our numerical simulation program is given in Figure 1. A detailed mathematical description of the model can be found elsewhere (Schmitt et al. 1995).

\subsection{Results}

By the hydrodynamic program, the different disk quantities needed as input parameters for the coagulation equation were determined. For several periods and disk radii, we calculated the mass distributions of coagulated grains confirming the power law dependences of these distributions, found by Mizuno et al. (1988), for wide mass ranges. As an example, Figure 2 shows the evolution of the dust mass distribution over a period of $10^{4}$ years for the outer radius $\left(6 \times 10^{14} \mathrm{~cm}\right)$ of the protoplanetary accretion disk. From these mass spectra, we determined the corresponding Rosseland mean dust opacities. In Figure 3, the Rosseland mean opacity is plotted as a function of the temperature for the initial mass spectrum and for spectra in timesteps of $10^{3} \mathrm{yrs}$ up to a total age of $10^{4} \mathrm{yrs}$. The opacity values and the steepness of the temperature dependence are lowered as coagulation proceeds. After a short period, the opacity decreases below the value where convective instability is possible. This means that the further evolution of the disk cannot be driven by opacity-induced convection. For a more general discussion of the influence of changes in the grain opacity 


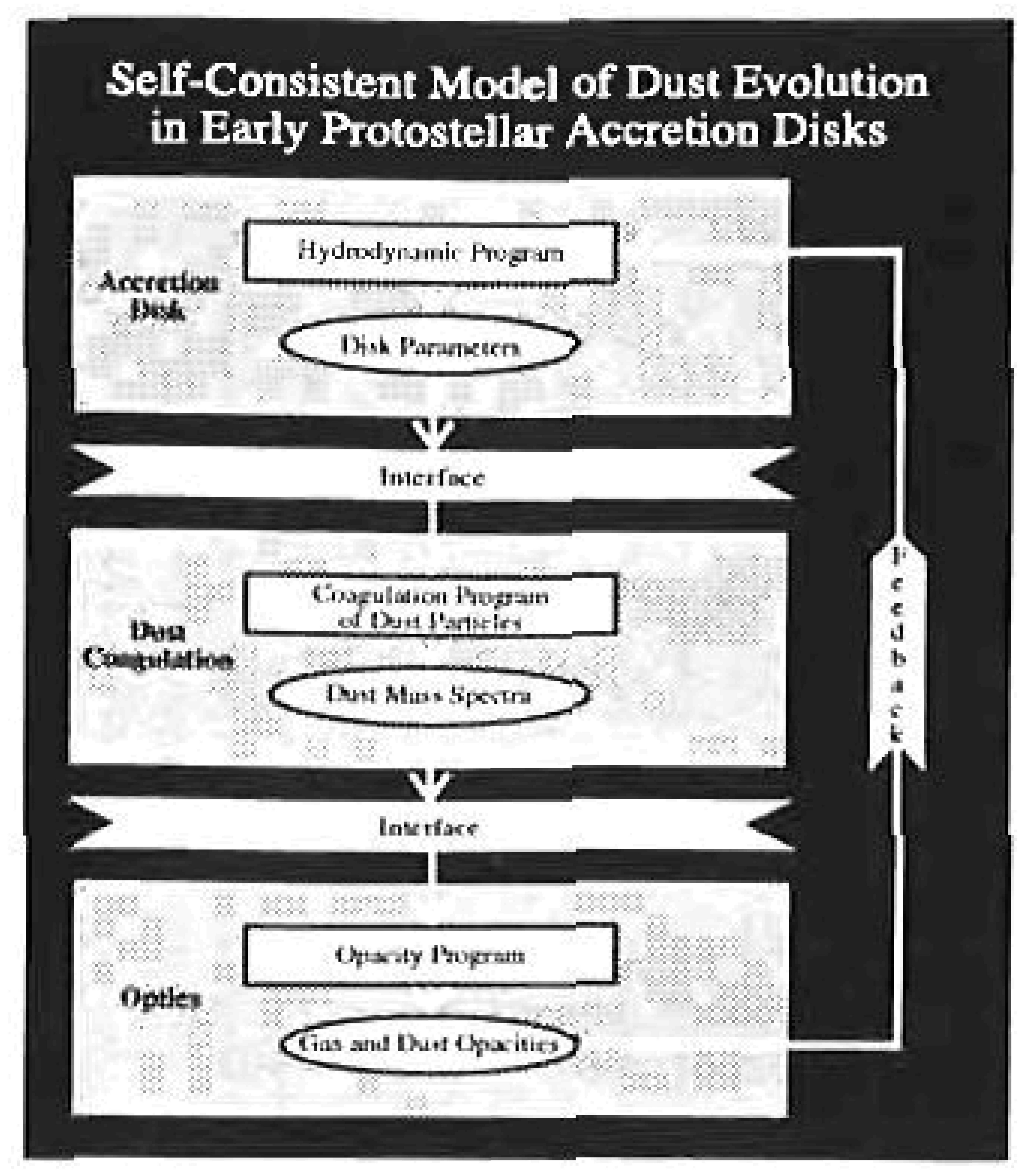

Figure 1. Scheme of the numerical simulation program for a self-consistent model of dust evolution in a protoplanetary accretion disk

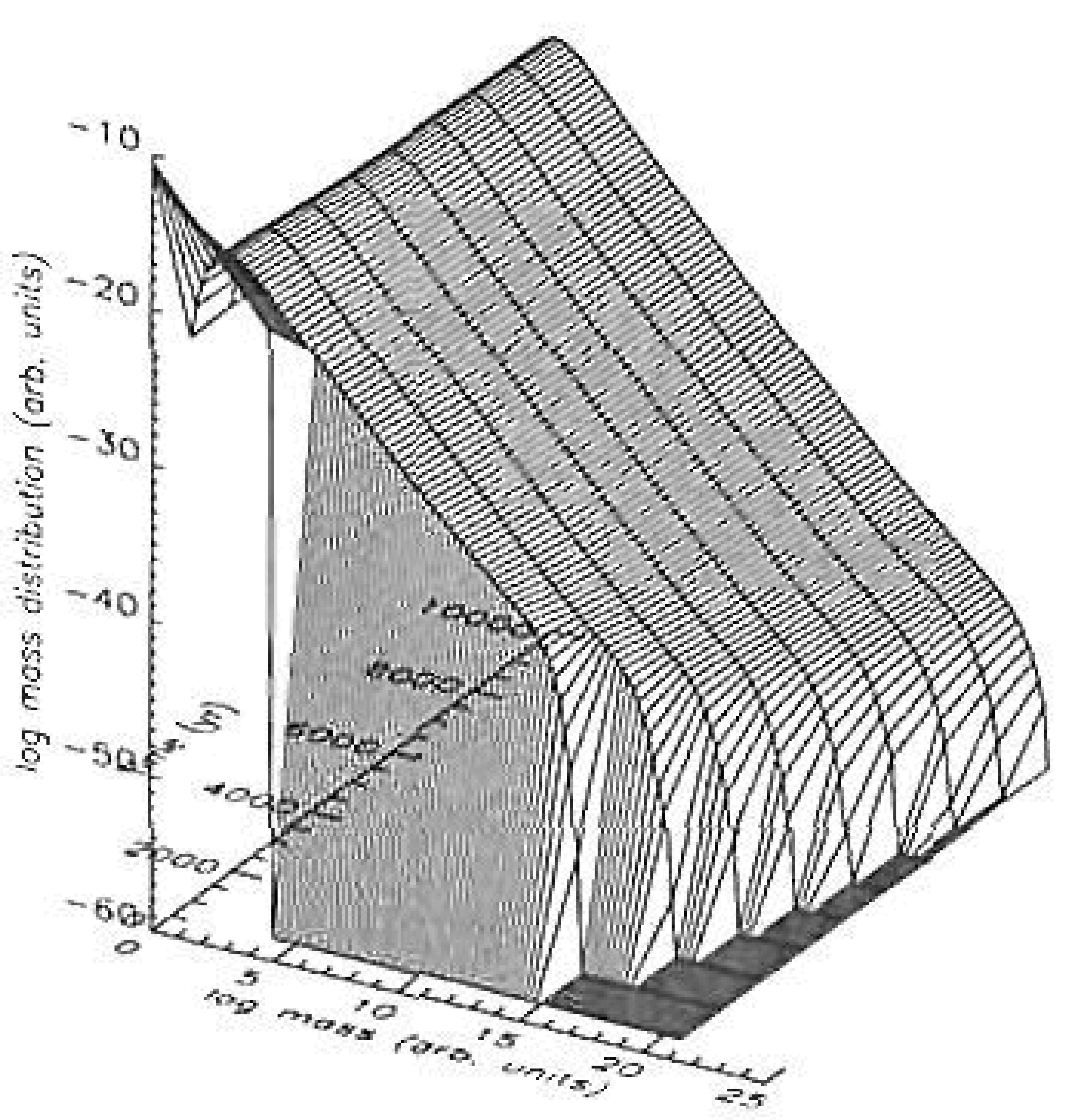

Figure 2. Evolution of the dust mass distribution over a period of $10^{4}$ years for the outer radius $(6 \times$ $10^{14} \mathrm{~cm}$ ) of the protoplanetary accretion disk

due to dust coagulation on the dynamical disk evolution, we refer to the paper by Schmitt et al. (1995).

\section{Grain Motion in a 2D Disk}

As the 1D radial model has no vertical structure, disk properties like temperature, density, and opacity are averaged over the total disk height. Vertical gas or dust velocities do not exist at all. On the other hand, "vertical" coagulation models are still 1D models. The radial transport of matter is neglected as it is the self-consistent time dependent disk evolution (see Weidenschilling 1992). Therefore, we are developing a $2 \mathrm{D}$ hydrodynamical code with dust transport and coagulation to get accurate values for the disk's local opacity. In this manner, we have a feedback to the hydrodynamical evolution. Starting with a two-dimensional radiation hydrodynamical code for accretion disks (see Kley 1989), we get a flowpattern of gas motion with inflow, outflow, and convective eddies. To this, we added a particle code which includes gravitational, inertial, and drag forces due to the gas. Hence, we are able to investigate relative velocities and migration processes for grains with different sizes and fractal dimensions. Figure 4 shows the ability of an eddy in the accretion disk to focus dust (disk parameters: $\alpha=10^{-2}$, mass accretion rate $=10^{-7} \mathrm{M}_{\odot} \mathrm{yr}^{-1}$ ). If the dust friction time is of the order of $\alpha^{2}$ times the overturn time of the eddy, efficient concentration of grains is possible. 


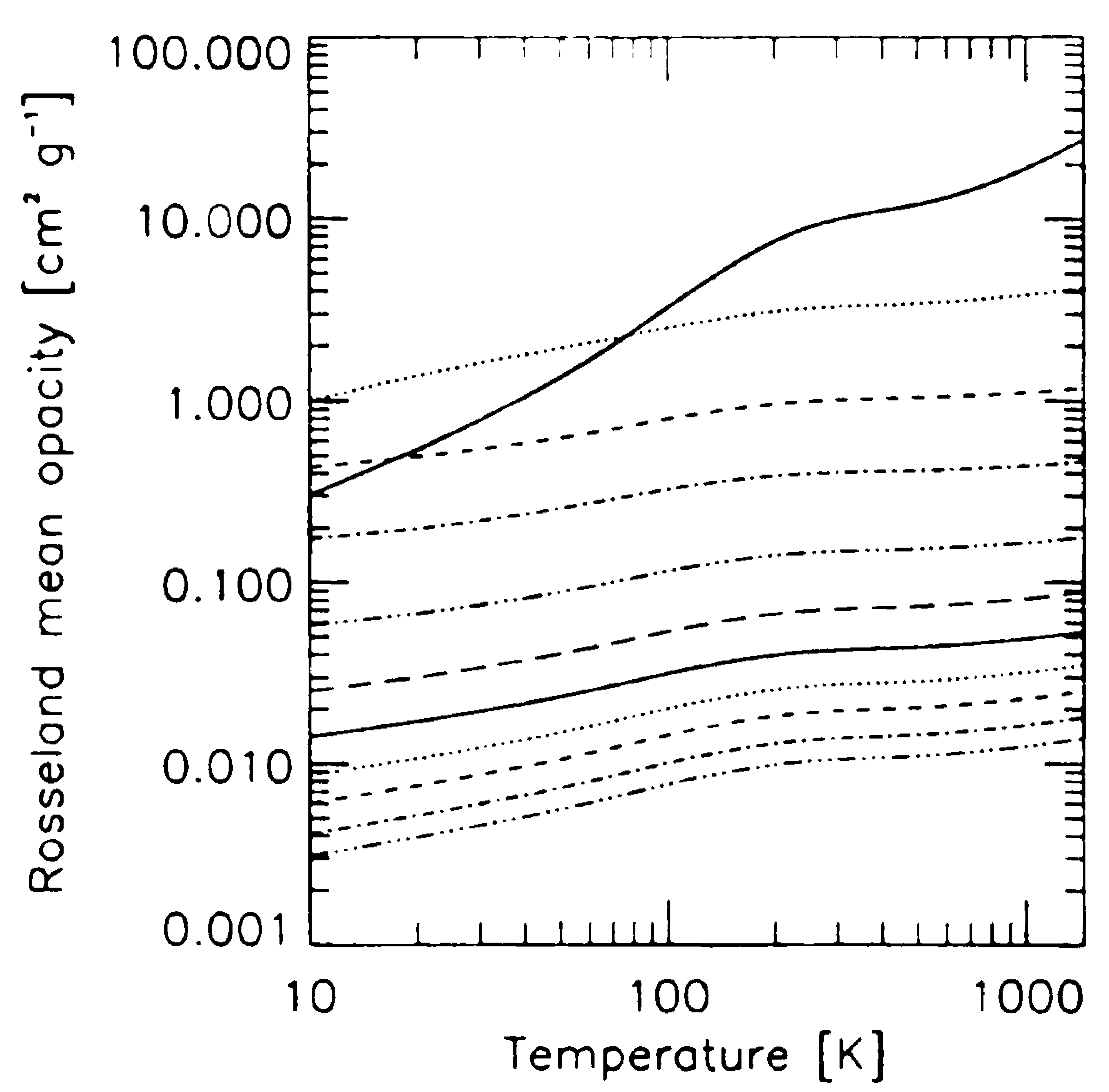

Figure 3. Temperature dependence of the Rosseland mean opacity for the initial mass spectrum (above; thick solid line) and for spectra after a period of $1,000, \ldots, 10,000$ years (below)

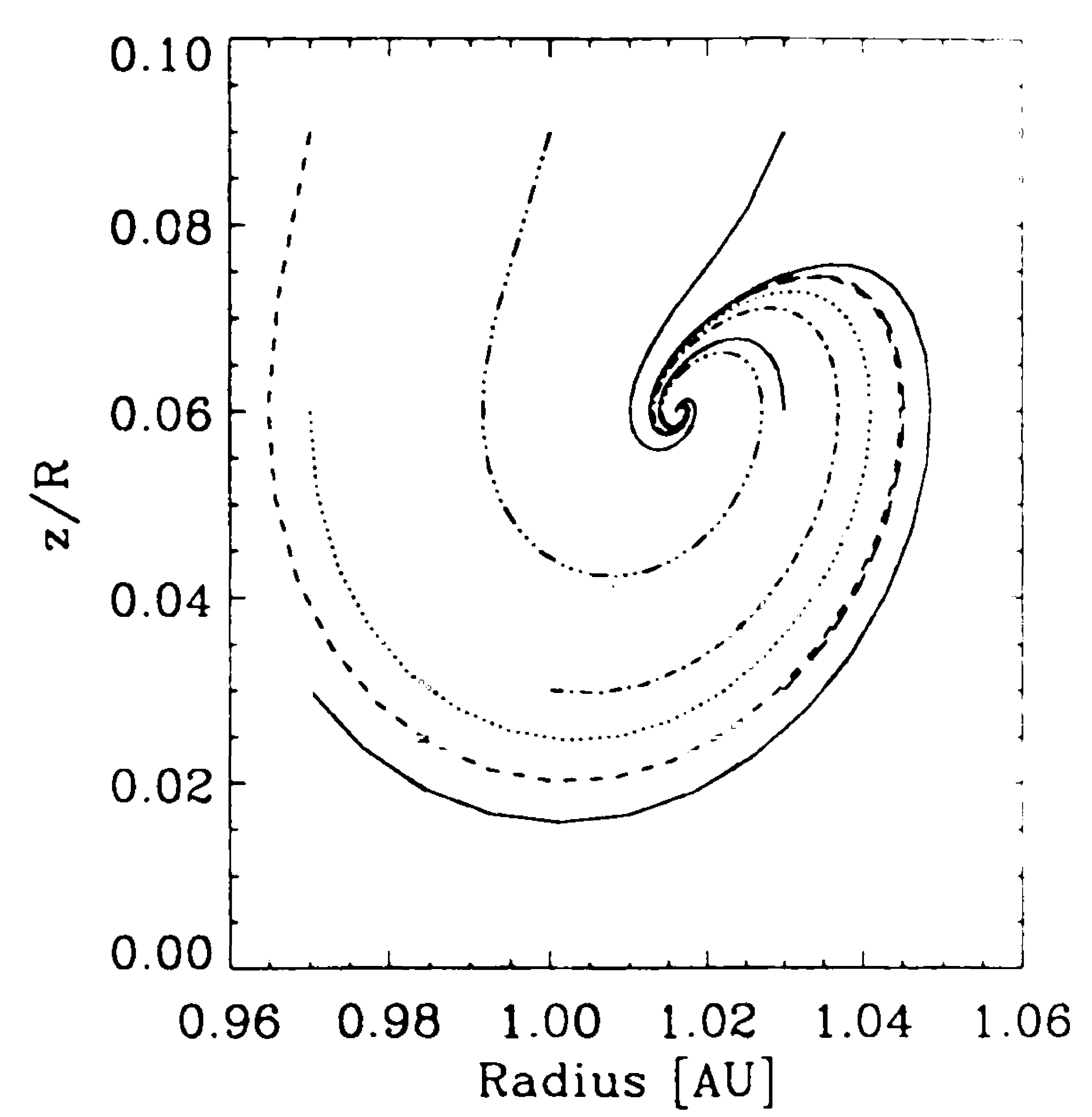

Figure 4. Tracks of 2 mm-sized dust grains in an eddy with its center at $R$ $=1 \mathrm{AU}$ at a half pressure scale height. The overturn time is $100 \mathrm{yr}$. The quantity $\mathrm{z}$ is the distance from the midplane

Acknowledgments. We thank K. R. Bell, W. Kley, V. Ossenkopf, and M. F. Sterzik for fruitful discussions and helpful suggestions.

\section{References}

Goldreich, P., \& Ward, W. R. 1973, ApJ, 183, 1051

Kley, W. 1989, A\&A, 208, 98

Mizuno, H. 1989, Icarus, 80, 189

Mizuno, H., Markiewicz, W. J., \& Völk, H. J 1988, A\&A, 195, 183

Ruden, S. P., \& Pollack, J. B. 1991, ApJ, 375, 740

Safronov, V. S. 1969, Evolution of the Protoplanetary Cloud and Formation of the Earth and Planets, Moscow: Nauka

Schmitt, W., Henning, Th., \& Mucha, R. 1995, in preparation

Völk, H., Jones, F., Morfill, G., \& Röser, S. 1980, A\&A, 85, 316

Weidenschilling, S. J. 1992, Planetesimal Formation 101, Summer School of Planetary Science

Weidenschilling, S. J., \& Cuzzi J N 1993, in Protostars and Planets III, E. H. Levy, J I. Lunine, Tucson: Univ Arizona, 1031 